\title{
The Impact of the E46K Mutation on the Properties of $\alpha$-Synuclein in Its Monomeric and Oligomeric States ${ }^{\dagger}$
}

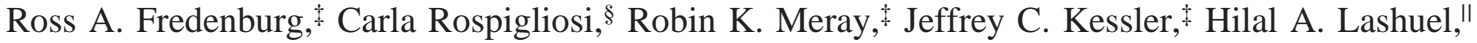 \\ David Eliezer, ${ }^{\S}$ and Peter T. Lansbury, Jr.*,
}

\begin{abstract}
Center for Neurologic Diseases, Brigham and Women's Hospital and Department of Neurology, Harvard Medical School, 65 Landsdowne Street, Cambridge, Massachusetts 02139, Department of Biochemistry and Program in Structural Biology, Weill Medical College of Cornell University, New York, New York 10021, and Laboratory of Molecular Neurobiology and Neuroproteomics, Brain Mind Institute, Ecole Polytechnique Federale de Lausanne, CH-1015 Lausanne, Switzerland
\end{abstract}

Received January 5, 2007; Revised Manuscript Received March 30, 2007

\begin{abstract}
The third and most recently identified Parkinson's disease-linked variant of the neuronal protein $\alpha$-synuclein to be identified (E46K) results in widespread brain pathology and early onset Parkinson symptoms (Zarranz et al. (2004) Ann. Neurol. 55, 164-173). Herein, we present biochemical and biophysical characterization of E46K $\alpha$-synuclein in various states of aggregation. Circular dichroism and nuclear magnetic resonance spectroscopy illustrate that the E46K mutation results in subtle changes in the conformation of the monomeric protein both free in solution and in the presence of SDS micelles. However, it does not alter the overall helical propensity of the protein in the presence of phospholipids. E46K $\alpha$-synuclein formed insoluble fibrils in vitro more rapidly than the wild type protein, and electron microscopy revealed that E46K $\alpha$-synuclein fibrils possess a typical amyloid ultrastructure. E46K $\alpha$-synuclein protofibrils, soluble aggregates that form during the transition from the monomeric form to the fibrillar form of $\alpha$-synuclein, were characterized by electron microscopy and gel filtration and were found to include annular species. The unique ability of a subfraction of E46K and wild type $\alpha$-synuclein protofibrils containing porelike species to permeabilize lipid vesicles was demonstrated in vitro using a real-time chromatographic method. In contrast to simplistic expectations, the total amount of protofibrils and the amount of permeabilizing activity per mole protein in the protofibril fraction were reduced by the E46K mutation. These results suggest that if the porelike activity of $\alpha$-synuclein is important for neurotoxicity, there must be factors in the neuronal cytoplasm that reverse the trends in the intrinsic properties of E46K versus WT $\alpha$-synuclein that are observed in vitro.
\end{abstract}

Parkinson's disease $\left(\mathrm{PD}^{1}\right)$ is a progressive neurodegenerative disorder characterized by resting tremor, bradykinesia, rigidity, and postural instability due to the selective loss of dopaminergic neurons within the substantia nigra $(2,3)$. While nearly all cases of PD are idiopathic, rare forms of autosomal dominant PD have been linked to the point mutations A53T (4), A30P (5), and E46K ( 1 ) in $\alpha$-synuclein, a presynaptic protein believed to be involved in synaptic vesicle trafficking $(6,7)$. Linkage between idiopathic PD and

\footnotetext{
$\dagger$ Financial support for these studies provided by NIH Grant AG08470 and a Morris K. Udall Parkinson's Disease Research Center of Excellence grant (NS038375) (to P.T.L.) and NIH Grant AG019391, the Irma T. Hirschl Foundation, and a gift from Herbert and Ann Siegel (to D.E.).

* Corresponding author. E-mail: plansbury@ rics.bwh.harvard.edu. Phone: (617) 768-8610. Fax: (617) 768-8606.

$\stackrel{+}{\ddagger}$ Harvard Medical School.

$\S$ Weill Medical College of Cornell University.

"Ecole Polytechnique Federale de Lausanne.

${ }^{1}$ Abbreviations: PD, Parkinson's disease; LB, Lewy bodies; EM, electron microscopy; WT, wild type; HBS, $10 \mathrm{mM}$ HEPES-NaOH/ $145 \mathrm{mM} \mathrm{KCl} / \mathrm{pH} 7.4$; CD, circular dichroism spectroscopy; PG, L- $\alpha-$ phosphatidyl-DL-glycerol; NMR, nuclear magnetic resonance spectroscopy; IPTG, isopropyl- $\beta$-D-thiogalactopyranoside; TBS/azide, $10 \mathrm{mM}$ Tris- $\mathrm{HCl} / 150 \mathrm{mM} \mathrm{NaCl} / 0.02 \%$ sodium azide/pH 7.4; HBS/EDTA, HBS/1 mM EDTA; ThioT, Thioflavin T; $\mathrm{HBS} / \mathrm{Ca}$, $\mathrm{HBS} / 5 \mathrm{mM} \mathrm{CaCl}_{2}$; SEC, size exclusion chromatography; FPD, familial PD.
}

$\alpha$-synuclein is suggested by the discovery that Lewy bodies (LB), intraneuronal cytoplasmic inclusions in the substantia nigra that are the pathological hallmark of $\mathrm{PD}$, are composed primarily of fibrillar $\alpha$-synuclein (8).

Monomeric $\alpha$-synuclein is natively unfolded in solution (9), but assumes $\beta$-sheet character as it aggregates through a series of intermediate, metastable oligomeric states (termed protofibrils) to a stable fibrillar conformation (10). In vitro studies have shown that both the A53T and A30P mutations in $\alpha$-synuclein alter the kinetics of fibrillization; the rate is increased for the A53T variant and retarded by the A30P substitution $(11,12)$. However, in both cases the rates of formation of prefibrillar oligomeric species are increased in comparison to wild type protein, suggesting a link between these protofibrils and disease (10). Initial studies involving E46K $\alpha$-synuclein indicate that the mutation enhances the formation of fibrillar species (13-15), but no examination of the formation of soluble aggregates has been reported.

Electron microscopy (EM) studies reveal that preparations of oligomeric $\alpha$-synuclein contain annular species that form as monomeric $\alpha$-synuclein progressively assembles into fibrils $(16,17)$. Indeed, $\alpha$-synuclein protofibrils, comprising either WT or the disease-associated variants, possess porelike activity and permeabilize negatively charged phos- 
pholipid vesicles in vitro $(18,19)$. This property is not observed with monomeric or fibrillar $\alpha$-synuclein. Studies in vivo support the theory that nonfibrillar aggregates of $\alpha$-synuclein are associated with neuronal pathology, as $\alpha$-synuclein-mediated toxicity in transgenic mice and Drosophila does not correlate with the appearance of fibrillar inclusions (20-22).

The identification of E46K $\alpha$-synuclein as the third pathogenic variant of the protein not only reinforces the strong connection between the protein and neurodegeneration; it also presents an opportunity to evaluate and refine current molecular models of $\alpha$-synuclein-mediated cytotoxicity. Previously, we proposed a model in which the formation of oligomeric protofibrils is linked to PD pathology (the toxic protofibril hypothesis) (23). Herein, we describe the biochemical and biophysical properties of E46K $\alpha$-synuclein, including structural characterization of the protein in its free and membrane-associated states, the aggregation dynamics of the variant in relation to wild type (WT) $\alpha$-synuclein, fibrillar and protofibrillar morphology, and the vesicle permeabilization activity of protofibrillar species. These results impact our current understanding of the toxic protofibril hypothesis.

\section{EXPERIMENTAL PROCEDURES}

Cloning, Expression, and Purification of $\alpha$-Synuclein Variants. E46K $\alpha$-synuclein mutant constructs were generated using oligonucleotide site-directed mutagenesis of previously generated wild type constructs (for NMR experiments) or using the megaprimer PCR method $(24,25)$ (for all other experiments), with the resulting PCR product ligated into the pT7-7 Escherichia coli expression vector (26). Isotopically labeled protein for NMR studies was expressed and purified using established protocols (27), and all proteins used in the other studies described were expressed as previously described (28) and isolated by the procedure below. Importantly, both protocols yield synuclein samples that give rise to identical NMR spectra (27). Cell lysis (Microfluidics Corporation M110-EHI) was followed by ammonium sulfate precipitation ( $30 \% \mathrm{w} / \mathrm{v}$ solution), anion exchange chromatography (Amersham Biosciences QSepharose) in $10 \mathrm{mM}$ Tris-HCl/1 mM EDTA/pH 8.0, and cation exchange chromatography (Amersham Biosciences S Sepharose) in $10 \mathrm{mM}$ sodium acetate $/ 1 \mathrm{mM}$ EDTA/pH 4.0. The final homogeneity of each preparation was determined by densitometric analysis of Coomassie-stained SDS-PAGE gel scans (NIH Image 1.61/ppc program). Purified preparations were lyophilized from ammonium bicarbonate buffer (100 mM) (buffer exchange achieved by Amersham Biosciences G25 chromatography) and stored at $-20{ }^{\circ} \mathrm{C}$ until use.

Size Exclusion Chromatography. Lyophilized samples of several variants including WT, E46K, and A53T $\alpha$-synuclein were resuspended in $10 \mathrm{mM}$ HEPES-NaOH/145 mM KCl/ pH 7.4 (HBS) to an approximate concentration of $200 \mu \mathrm{M}$. Insoluble material was removed by $0.2 \mu \mathrm{m}$ filtration (Millipore) prior to injection of each $\alpha$-synuclein preparation onto a $24 \mathrm{~mL}$ Superose 6 gel filtration column (Amersham Biosciences) previously equilibrated in HBS. The eluate, passed through the column at $0.5 \mathrm{~mL} / \mathrm{min}$, was monitored with a Waters 996 PDA detector (Waters Corporation).
Retention times were determined using Waters Millennium data processing software (Waters Corporation).

Circular Dichroism Spectroscopy (CD). WT and E46K $\alpha$-synuclein protein solutions $(60 \mu \mathrm{M})$ were each prepared in distilled water or distilled water containing various concentrations of L- $\alpha$-phosphatidyl-DL-glycerol (PG) vesicles in $1 \mathrm{mM}$ HEPES-NaOH/14.5 mM KCl/0.1 mM EDTA/pH 7.4. Far-UV CD spectra were obtained in a $0.1 \mathrm{~cm}$ cuvette using an Aviv 62A DS spectropolarimeter at $25{ }^{\circ} \mathrm{C}$. Measurements were made at $1 \mathrm{~nm}$ intervals with 5-10 s response times, and the final curves for each sample represent the mean of three separate scans.

Nuclear Magnetic Resonance (NMR) Spectroscopy. Samples for NMR experiments were made to $\sim 140 \mu \mathrm{M}$ protein concentration. For the free state, lyophilized protein was dissolved in sample buffer $\left(100 \mathrm{mM} \mathrm{NaCl}, 10 \mathrm{mM} \mathrm{Na}_{2} \mathrm{HPO}_{4}\right.$, $\mathrm{pH} 7.4$ in $90 \% / 10 \% \mathrm{H}_{2} \mathrm{O} / \mathrm{D}_{2} \mathrm{O}$ ) followed by the removal of any residual large-scale aggregates using Microcon YM-100 centrifugal filters (Millipore). Samples containing SDS micelles were prepared by dissolving the protein in sample buffer containing $40 \mathrm{mM}$ SDS. All NMR experiments were performed on a $600 \mathrm{MHz}$ Varian INOVA spectrometer, at a sample temperature of either $10{ }^{\circ} \mathrm{C}$ (free state) or $40{ }^{\circ} \mathrm{C}$ (SDS micelles bound state). Spectra were recorded in succession from matched wild type and E46K samples. Data were processed with NMRPipe (29) and analyzed using NMRView (30). Spectra were referenced indirectly to DSS and ammonia (31) using the known chemical shift of water. Resonances for the mutant protein were assigned by inspection and comparison with the previously assigned spectra of the wild type protein, and are therefore tentative in some cases, though for many resonances the assignments could be made confidently.

Preparation of $\alpha$-Synuclein Solutions for Fibrillization Studies. Lyophilized protein samples were dissolved in $10 \mathrm{mM}$ Tris- $\mathrm{HCl} / 150 \mathrm{mM} \mathrm{NaCl} / \mathrm{pH} 7.4$ with $0.02 \%$ sodium azide (TBS/azide) to achieve an approximate concentration of $4 \mathrm{mg} / \mathrm{mL}$. Successive filtration through $0.2 \mu \mathrm{m}$ filter units (Millipore) and $100 \mathrm{kDa}$ MWCO filter units (Millipore) was performed to prepare homogeneous, monomeric protein solutions. The total protein concentration of each filtrate solution was determined by UV absorbance $(280 \mathrm{~nm})$, BCA assay (Pierce), and/or quantitative amino acid analysis. Samples were diluted to a total protein concentration of 70 $\mu \mathrm{M}$ in a total volume of $1.0 \mathrm{~mL}$ of TBS/azide in $1.5 \mathrm{~mL}$ polypropylene centrifuge tubes. The samples were incubated at $37{ }^{\circ} \mathrm{C}$ in a tissue culture roller drum (New Brunswick Scientific Co., model TC-7) rotating at $43 \mathrm{rpm}$. Aliquots were removed from the roller drum at $4 \mathrm{~h}$ intervals for time point sampling.

Thioflavin T Fluorescence Assay for Fibril Formation. Aliquots of the $\alpha$-synuclein incubations were assayed in triplicate at a concentration of $5 \mu \mathrm{M}$ by Thioflavin $\mathrm{T}$ (ThioT) fluorescence as described previously (32).

Gel Filtration and UV Absorbance Measurements of Fibrillization Samples. Relative monomer concentrations within fibrillization time point samples were determined by monitoring the UV absorption at $280 \mathrm{~nm}$ of the effluent from a Shodex KW-G column (Showa Denko) as described previously (28). Peak areas were integrated using Waters Millennium Software (Waters Corp). 
Electron Microscopy. For protofibril morphology evaluation, the oligomeric fraction of a protofibril-enriched $\alpha$-synuclein preparation was separated from the monomeric fraction using a Superdex 200 gel filtration column (Amersham Biosciences) run in HBS/EDTA, and pools were concentrated using $10 \mathrm{kDa}$ MWCO centrifuge filter units (Millipore). For analysis of fibril morphology, insoluble material generated during a typical fibrillization study (as described above) was isolated by centrifugation at $12000 \mathrm{~g}$ for $5 \mathrm{~min}$. Resulting pellets were resuspended in $150 \mu \mathrm{L}$ of $10 \mathrm{mM}$ Tris- $\mathrm{HCl} / \mathrm{pH}$ 8.0. Each sample was coated neat on a Formvar- and carbon-coated copper grid and washed with two drops of distilled water and one drop of uranyl acetate $(6 \mathrm{mg} / \mathrm{mL})$. The grids were then stained with uranyl acetate $(6 \mathrm{mg} / \mathrm{mL})$ for $1 \mathrm{~min}$. The samples were then studied in an $80 \mathrm{kV}$ Jeol-1200EX electron microscope; protofibril samples were examined at a magnification of $25000-30000 \times$ and fibril samples at $10000-60000 \times$. The grids were thoroughly examined, and representative samples of the structures present in the sample were recorded on film.

Preparation of $\alpha$-Synuclein Oligomeric Species. As previously reported, soluble aggregates of $\alpha$-synuclein can be isolated from a solution resulting from the resuspension of lyophilized $\alpha$-synuclein protein $(16,18)$. To achieve consistently high and reproducible yields of these oligomeric species, the following changes to the established protocol were introduced. Lyophilized $\alpha$-synuclein protein was resuspended in ammonium bicarbonate buffer (100 mM), diluted in the same buffer to a set concentration (typically $200 \mu \mathrm{M}$ ), and then flash frozen in liquid nitrogen in a fully upright position before relyophilization. This procedure ensured that each preparation presented the same exposed surface area during lyophilization in order to minimize differences in the rate of sublimation between samples. The dehydrated powder was resuspended to $400 \mu \mathrm{M}$ final concentration in $10 \mathrm{mM}$ HEPES-NaOH/145 mM KCl/1 mM EDTA/pH 7.4 (HBS/EDTA). Insoluble aggregates were removed by $0.2 \mu \mathrm{m}$ filtration, and preparations were stored at $4{ }^{\circ} \mathrm{C}$ for no more than one week before analysis.

Synthetic Vesicle Permeabilization. Activity measurements of $\alpha$-synuclein protofibril fractions were obtained by an online permeabilization method developed by Volles (33), with minor modifications. In brief, PG vesicles loaded with the calcium-sensitive fluorescent dye fura- 2 were created as described previously (19) and diluted to approximately $0.1 \mathrm{mg} / \mathrm{mL}$ (Note: the precise dilution of each vesicle preparation was determined empirically so that each preparation yielded a baseline fluorescence of $500 \mathrm{RFU}$ ) in $10 \mathrm{mM}$ HEPES-NaOH/145 mM KCl/5 mM CaCl $2 / \mathrm{pH} 7.4$ (HBS/Ca). Protofibril-enriched $\alpha$-synuclein solutions were resolved using a $24 \mathrm{~mL}$ Superose 6 column (Amersham Bioscience) equilibrated in HBS (no calcium). The effluent from the column (at a flow rate of $0.5 \mathrm{~mL} / \mathrm{min}$ ) was passed through a spectrophotometer (Waters PDA 996) and then was combined with the vesicle solution delivered by syringe pump (at a flow rate of $0.1 \mathrm{~mL} / \mathrm{min})$. After $0.6 \mathrm{~mL}(1 \mathrm{~min})$ of tubing in which to interact, the protein/vesicle mixture was passed through a scanning fluorescence detector (Waters model 474) to measure the fluorescence increase at $500 \mathrm{~nm}$ (334 nm excitation, $40 \mathrm{~nm}$ bandwidth) upon exposure of fura-2 to $\mathrm{Ca}^{2+}$. Molar permeabilization activity of each quaternary structural fraction was determined by integration of the peak of $280 \mathrm{~nm}$ absorbance (22.5 min retention time) and the peak of $500 \mathrm{~nm}$ fluorescence (23 min retention time) (Waters Millenium software).

\section{RESULTS}

Monomeric E46K and WT $\alpha$-Synuclein Share Similar, but Not Identical, Physical Properties. The E46K variant of $\alpha$-synuclein displays gross solubility and electrostatic properties similar to those of WT $\alpha$-synuclein, as identical purification protocols are sufficient to achieve a similar purity level and yield for each variant. SDS-PAGE analysis indicates that, like WT $\alpha$-synuclein (data not shown), the E46K variant can be isolated to $>95 \%$ homogeneity (Supporting Information Figure 1). However, the retention time of E46K $\alpha$-synuclein upon size exclusion chromatography (SEC) analysis is increased in comparison to that of WT; this is contrasted by the highly similar retention times of the WT protein and another pathogenic $\alpha$-synuclein variant, A53T (Figure 1A). When analysis of variance testing is applied to the mean retention times of the three variants, a significant difference is detected $(F(2,40)=14.16, p=$ $2 \times 10^{-5}$ ); Tukey's HSD pairwise comparison procedure indicates that the differences in retention time between WT and E46K $\alpha$-synuclein and A53T and E46K $\alpha$-synuclein are statistically significant. Monomeric $\alpha$-synuclein is known to elute from SEC at an apparent molecular weight much higher than its sequence-defined mass of $14460 \mathrm{kDa}$; this discrepancy may reflect the effect on Stokes radius of the protein's extended random coil conformation (ref 9, Figure 1B). The increased retention time of E46K may therefore indicate that the variant exists in solution in a slightly more compact form than the WT protein. Although it is possible that the difference in net charge between WT and E46K $\alpha$-synuclein results in a nonspecific ion-exchange interaction with the column matrix, the probability of such an effect is minimized by the ionic strength of the mobile phase (which contains $145 \mathrm{mM} \mathrm{KCl}$ ).

$\mathrm{CD}$ analysis indicates a similar random coil secondary structure for both E46K and WT $\alpha$-synuclein when free in solution (Figure 1B). In the presence of acidic phospholipid vesicles, both E46K and WT $\alpha$-synuclein undergo a structural transition to a primarily $\alpha$-helical state, indicating that the mutation does not disrupt this known property of $\alpha$-synuclein $(27,34)$. This conversion from random coil to $\alpha$-helix occurs in a manner dependent on lipid concentration, and no evidence for a mutation-linked alteration of membrane affinity was revealed by these experiments, as both WT and E46K $\alpha$-synuclein displayed highly similar concentration dependencies (Supporting Information Figure 2).

E46K Mutation of $\alpha$-Synuclein Results in Subtle Changes in Protein Conformation. We further examined the conformation of monomeric $\alpha$-synuclein in solution using NMR spectroscopy, as chemical shifts of nuclei are sensitive probes of local structure and chemical shift changes are highly sensitive indicators of structural alterations. The proton-nitrogen correlation (HSQC) spectrum of E46K mutant $\alpha$-synuclein overlaid with that of the WT protein (Figure 2A) shows that residues close to the mutation point (Lys43-Val49) shift noticeably with respect to WT, as would be expected from the local change caused by the replacement of the glutamate side chain with a lysine, but that the 

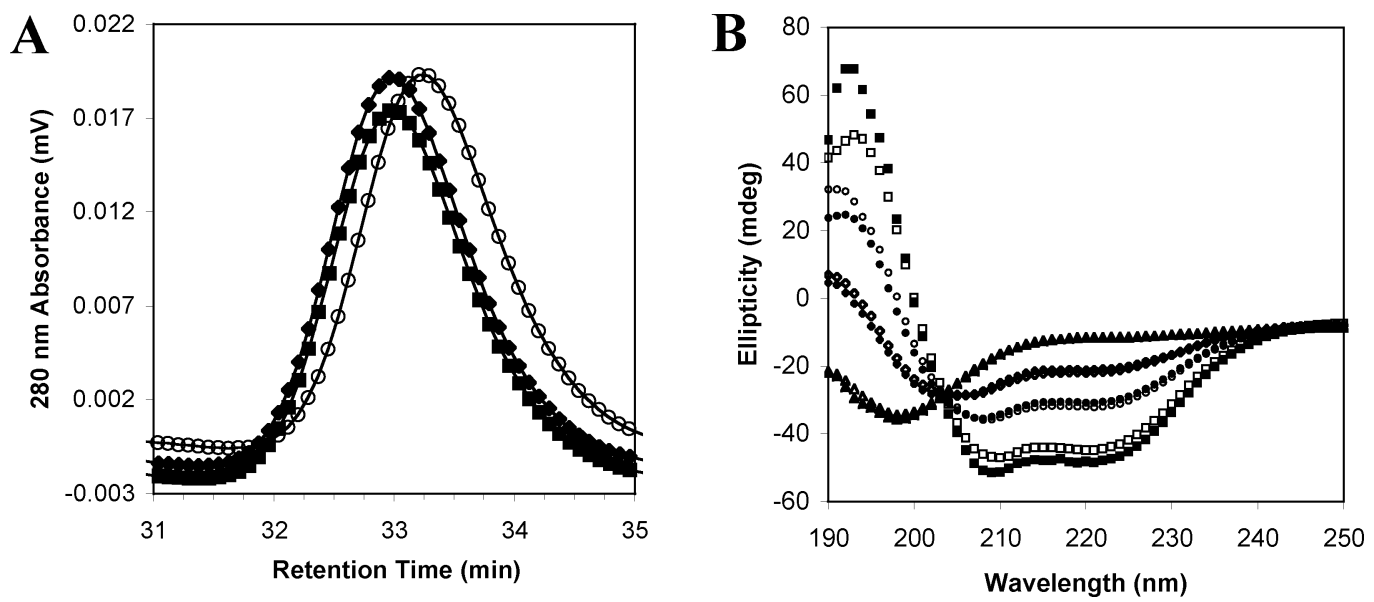

FIGURE 1: (A) Overlaid chromatograms displaying absorbance at $280 \mathrm{~nm}$ of solutions of WT (filled diamonds), E46K (open circles), and A53T (filled squares) monomeric $\alpha$-synuclein eluted from a Superose 6 size exclusion column. (B) CD spectra of purified WT and E46K $\alpha$-synuclein, in the presence of various concentrations of lipids. In isolation, both WT (open triangles) and E46K (filled triangles) assume a random coil confirmation (single minimum at $198 \mathrm{~nm}$ ). In the presence of $2 \mathrm{mg} / \mathrm{mL}$ PG vesicles, both WT (open squares) and E46K (filled squares) assume primarily $\alpha$-helical secondary structure (two minima at 209 and $221 \mathrm{~nm}$ ). Also shown are solutions of WT and E46K $\alpha$-synuclein in the presence of $0.5 \mathrm{mg} / \mathrm{mL}$ PG vesicles (open and filled circles, respectively) and $0.25 \mathrm{mg} / \mathrm{mL}$ PG vesicles (open and filled diamonds, respectively).

remainder of the spectrum appears largely unperturbed. To further quantitate the spectral changes, the differences in the weighted average of the amide proton and nitrogen chemical shifts between the WT and mutant protein were calculated and are plotted in Figure 2B. The data indicate that there are no significant chemical shift perturbations away from the site of the mutation. There were also no significant changes in relative peak heights between the WT and mutant spectra (data not shown).

In the presence of SDS micelles, the N-terminal lipidbinding domain of $\alpha$-synuclein adopts a structure consisting of two helical segments that has been extensively characterized using NMR for WT $(27,35-37)$ as well as the A30P and A53T mutants $(38,39)$. A comparison of protonnitrogen correlation spectra of the E46K and wild type proteins (Figure 3A) reveals that while many resonances in the previously assigned (35) wild type spectrum precisely overlap equivalent resonances in the spectrum of the mutant, a significant number of resonances are shifted in the presence of the mutation. The clearest shifts occur for residues near the mutation site (for example G41, S42, T44, G51, V52, T54, and A56), as expected, many of which lie in the linker region between the two micelle-bound helices. It is not possible, based on these spectra, to assess whether these changes occur only as a consequence of the change in the local chemical environment caused by the presence of the mutation, or whether they also reflect local structural changes. In addition to local changes, however, many resonances originating far from the site of mutation also shift noticeably, including A19, E20, T22, V26, and A27, which are located in the first helix of the micelle-bound protein, and V63, G68, A69, V74, and G84, which are located in the second helix. These chemical shift changes are not large enough to indicate whole-scale alteration of the secondary structure of the protein (this would be expected to result in shift changes of $1 \mathrm{ppm}$ or greater), and it is thus likely that the two helices of the micelle-bound protein remain intact. Nevertheless, there is clearly some change that occurs in the environment of both helices as a result of the mutation, which itself falls in the linker region.
Because the mutation-induced chemical shift changes for the SDS-bound state are more substantial and widespread than those observed for the free state, some resonance assignments could not be transferred in a straightforward manner from the wild type to the mutant spectrum, and at present no independent assignments are available for the SDS-bound E46K mutant. Therefore, to quantitate the chemical shift changes between the wild type and mutant proteins, we used the following strategy. Each wild type resonance assignment was transferred to the nearest resonance in the mutant protein spectrum, unless that resonance was already assigned to a different (nearer) resonance, in which case the next nearest resonance was selected. This strategy is not foolproof, as resonances may shift unpredictably and can even, for example, trade places in principle. Nevertheless, in general this strategy will underestimate the magnitude of the actual chemical shift change experienced by any given resonance. Thus, a plot of the weighted average of the thus estimated amide proton and nitrogen chemical shift changes between the WT and mutant spectra should allow for a reliable assessment of which regions of the protein are affected by the mutation. Such a plot, shown in Figure 3B, confirms that significant chemical shift changes take place at sites distant from the E46K mutation, with effects ranging approximately from residues 20 to 74 . This can be contrasted with the situation in the free state (Figure 2B), where only local changes are observed.

E46K $\alpha$-Synuclein Forms Fibrils More Rapidly Than WT $\alpha$-Synuclein. The formation of insoluble amyloid fibrils from monomeric $\alpha$-synuclein solutions is a well-documented phenomenon, and the propensity of a particular variant of $\alpha$-synuclein to form fibrils in vitro may relate to the appearance of fibrillar deposits in the human brain. To measure the incorporation of monomeric $\alpha$-synuclein into fibrils, we exploited the binding affinity of the fluorescent dye ThioT for amyloid fibrils, as well as the altered solubility characteristics of $\alpha$-synuclein upon conversion to the fibrillar form. Solutions of WT, E46K, or a 1:1 mixture of both $\alpha$-synuclein variants display loss of soluble monomer and appearance of ThioT-reactive species upon incubation with 

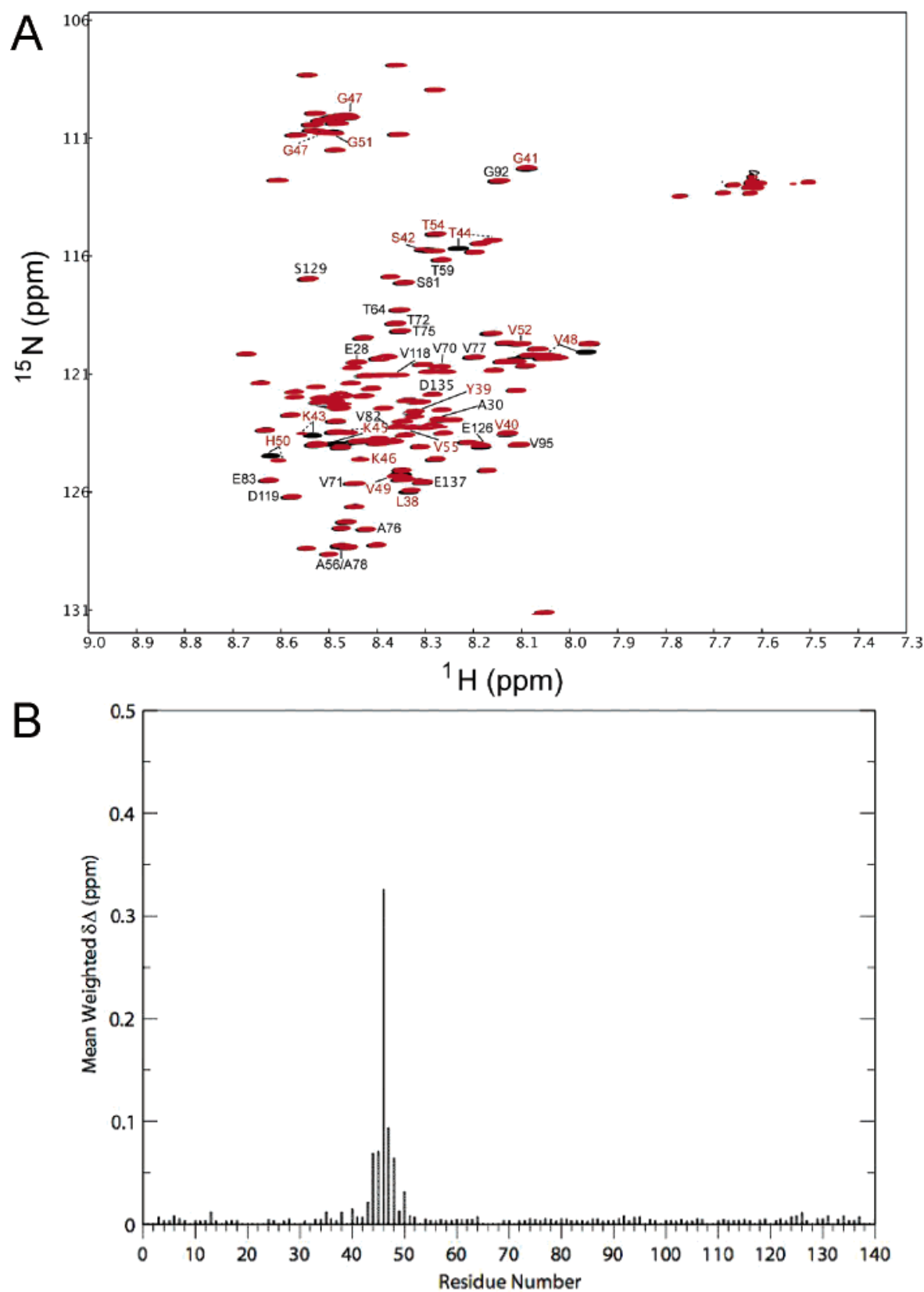

FIGURE 2: (A) Overlaid contour plots of the ${ }^{1} \mathrm{H}-{ }^{15} \mathrm{~N}$ HSQC spectra of WT (black) and E46K (red) $\alpha$-synuclein in the free state. Amino acid residues that shift with respect to the wild type protein are labeled. Labels in red correspond to \pm 9 amino acid residues from the mutation point. Residues that are further away are in black. (B) Mean weighted ${ }^{1} \mathrm{H}-{ }^{15} \mathrm{~N}$ chemical shift differences $\left[\mathrm{calculated}\right.$ as $\left[\left(\Delta \delta^{1} \mathrm{H}\right)^{2}\right.$ $\left.\left.+\left(\Delta \delta^{15} \mathrm{~N}\right)^{2} / 25\right]^{1 / 2} / 2(58)\right]$ between WT and E46K $\alpha$-synuclein in the free state.

agitation for $16 \mathrm{~h}$ as fibrils form in a nucleation-dependent manner (Figure 4). The E46K variant forms fibrils most readily, with a lag time of approximately $8 \mathrm{~h}$. A previous report established that the rate of $\mathrm{E} 46 \mathrm{~K} \alpha$-synuclein fibril formation is similar to that of the A53T variant (13). WT $\alpha$-synuclein is considerably slower to form fibrils, with a lag time of approximately $12 \mathrm{~h}$. A 1:1 mixture of WT and E46K (representative of the heterozygous condition in patients) is intermediate in fibrillization propensity. For each sample, enhancement of ThioT fluorescence appears at time points corresponding to the initial loss of monomer, indicating incorporation of monomeric $\alpha$-synuclein into fibrils (Figure 4 inset). The fibrils formed by E46K $\alpha$-synuclein during the assay were analyzed by EM, and are morphologically similar to WT $\alpha$-synuclein fibrils (Figure 5). The mutant and WT fibrils are each approximately $10 \mathrm{~nm}$ in width, are unbranched and appear rigid, and tend to associate into paired helical filaments. These properties are consistent with those of fibrillar $\alpha$-synuclein isolated from diseased human brain as well as with the defining characteristics of the broad class of amyloid proteins (32).

E46K and WT $\alpha$-Synuclein Form Annular Protofibrils. In addition to fibrils, $\alpha$-synuclein aggregation results in a variety of soluble oligomeric species that seem to serve as fibrillization intermediates, or protofibrils $(10,16)$. In order to examine the effects of the E46K mutation on the formation of protofibrils, $\alpha$-synuclein aggregates generated via lyophilization were separated from the monomeric species and concentrated to facilitate analysis of the structural composition of the mixture of oligomeric forms. Figure 6A illustrates E46K $\alpha$-synuclein aggregate composition, and highlights the formation of small $(10-15 \mathrm{~nm})$, distinct, and regular 

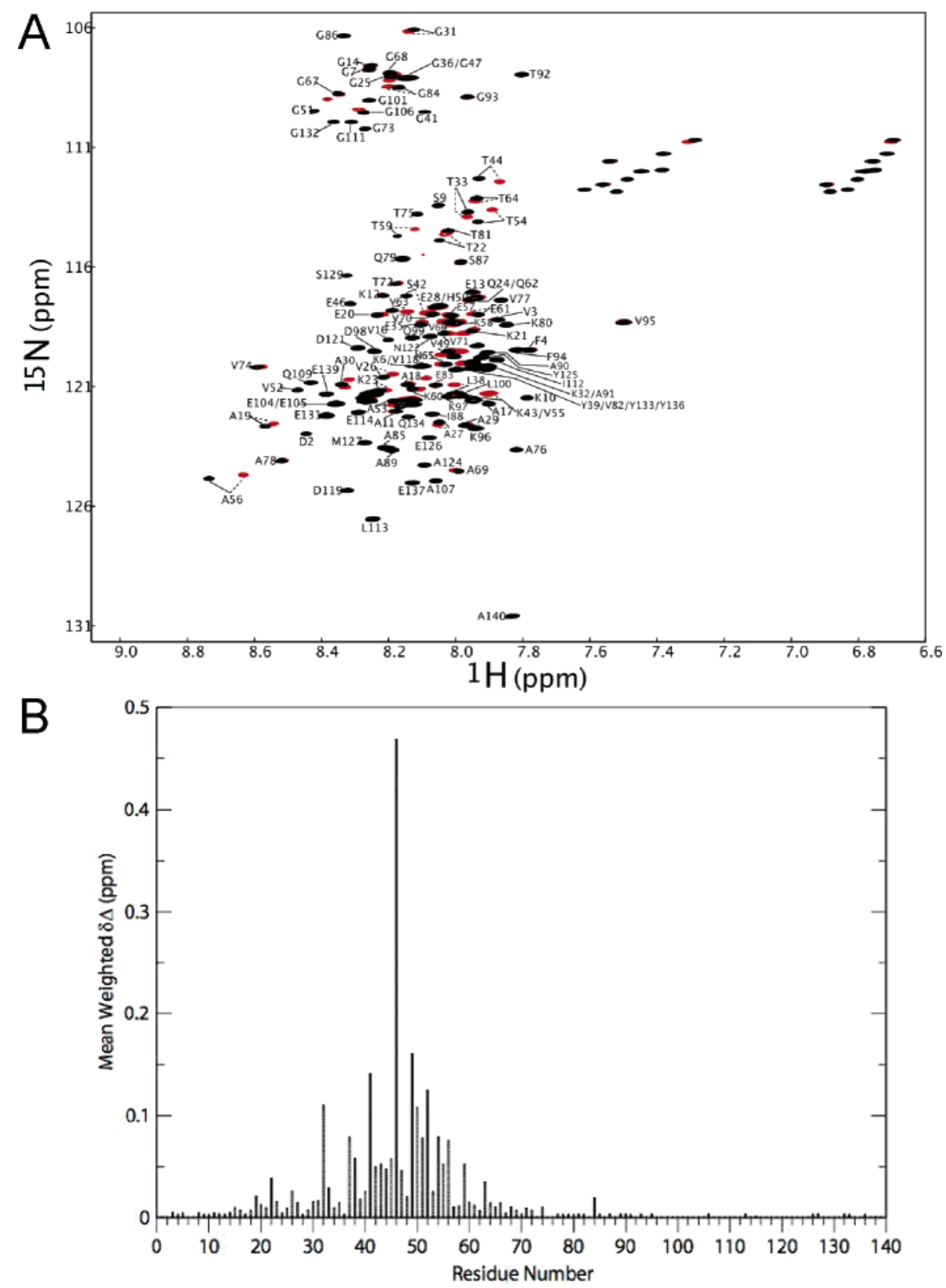

FIGURE 3: (A) Overlaid contour plots of the ${ }^{1} \mathrm{H}-{ }^{15} \mathrm{~N}$ HSQC spectra of WT (black) and E46K (red) in the SDS micelle-bound state. Resonance assignments of the wild type protein are denoted, and for resonances that shift in the mutant spectrum the location of the resonance used to calculate the chemical shift difference upon mutation, as described in the text, is noted. (B) Mean weighted ${ }^{1} \mathrm{H}-{ }^{15} \mathrm{~N}$ chemical shift differences $\left[\left[\left(\Delta \delta^{1} \mathrm{H}\right)^{2}+\left(\Delta \delta^{15} \mathrm{~N}\right)^{2} / 25\right]^{1 / 2} / 2(57)\right]$ between WT and E46K $\alpha$-synuclein in the SDS-bound state, calculated as described in the text.

elements, many of which appear to be annular in nature. WT $\alpha$-synuclein also generates numerous annular aggregates of similar conformation to the E46K species, with diameters ranging from 11 to $17 \mathrm{~nm}$ (Figure 6B). The conformation and size of the porelike oligomeric structures presented here are consistent with previous reports of $\alpha$-synuclein aggregate formation $(16,40)$, and no significant difference in the average outer diameter of WT and E46K $\alpha$-synuclein annular protofibrils was revealed upon examination of images from several independent experiments (data not shown). It is important to note that these images serve to establish the structural properties of individual oligomeric species formed from each $\alpha$-synuclein variant, and do not illustrate a quantitative difference in the prevalence of annular protofibrils among the broad array of aggregate forms generated by each variant. In fact, the apparent difference in the abundance of larger soluble oligomeric species between E46K and WT $\alpha$-synuclein in Figure 6 was examined but not substantiated by quantitative SEC analysis (data not shown).

Permeabilizing Activity Is Confined to a Specific Subset of $\alpha$-Synuclein Oligomeric Species. The ability of $\alpha$-synuclein protofibrils to disrupt synthetic phospholipid vesicles has been extensively characterized $(18,19)$ and suggests a potential mechanism of direct $\alpha$-synuclein-mediated cytotoxicity. The development of an online permeabilization assay (33) has facilitated the comparison of the vesicle permeabilizing activity of E46K and WT $\alpha$-synuclein aggregates by reducing the time delay between isolation and activity measurement, and by eliminating the need for separate purification, filtration, and concentration determination steps. Such processing steps can allow re-equilibration of the protein mixture, thereby altering the distribution of 


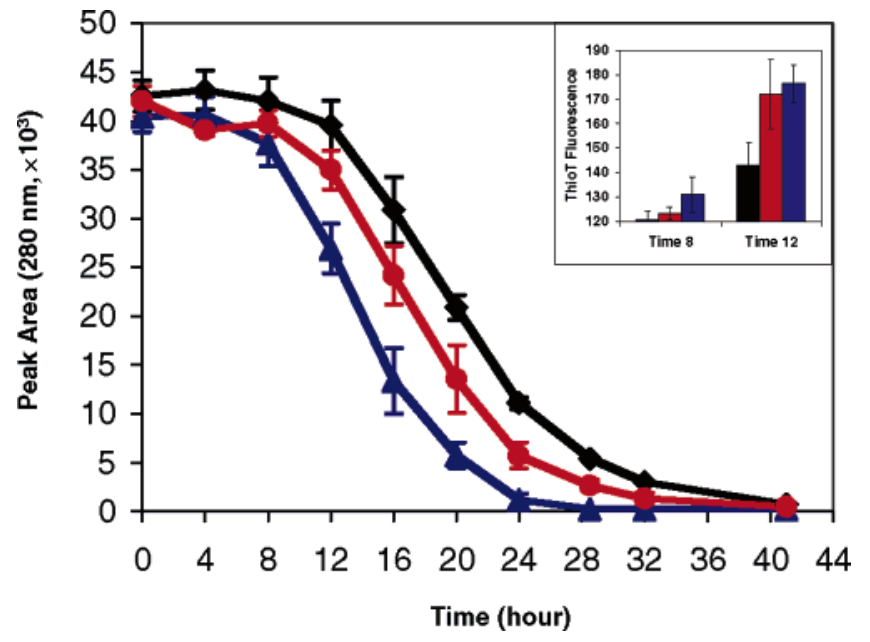

FIGURE 4: Fibrillization kinetics of pure samples of WT $\alpha$-synuclein, E46K $\alpha$-synuclein, and an equimolar mixture of both. All samples were examined at $70 \mu \mathrm{M}$ total protein concentration, with $43 \mathrm{rpm}$ rolling agitation. WT, black diamonds; E46K, blue triangles; WT:E46K (1:1), red circles. Inset graph displays ThioT reactivity at early time points, confirming that fibrils form as monomeric $\alpha$-synuclein is removed from the solution. Data presented are representative of three independent experiments (see also Supporting Information Figure 4). Error bars represent plus or minus one standard error of triplicate samples.

oligomeric species (data not shown). Molar activity measurements can be obtained from a single SEC eluate flow path by determining the protein abundance at a given retention time and the corresponding peak of vesicle permeabilization (Figure 7). In addition, the simultaneous monitoring of the protein quaternary structure distribution and permeabilizing activity of $\alpha$-synuclein preparations reveals information about the nature of the active species. Figure 7 (blue trace) illustrates the separation achieved by the Superose 6 SEC column. The sharp peak at 15 min (termed Void) occurs at the void volume of the column, and contains soluble aggregates of $>40000 \mathrm{kDa}$ molecular mass. The broad peak at $22.5 \mathrm{~min}$ (termed Protofibril) contains smaller soluble aggregates, including annular protofibrils (Supporting Information Figure 3). The peak at 30 min (termed Dimer) is composed of the dimeric form of $\alpha$-synuclein, and the large peak at 33 min (termed Monomer) contains the monomeric form. When the protein within each of these peaks interacts with vesicles containing the calcium-sensitive fluorescent dye fura-2, a fluorescence signal is monitored to detect exposure of the vesicle interior to the calcium-containing mobile phase (Figure 7, red trace). To compare the activity levels of the different aggregate forms of $\alpha$-synuclein, molar activity values were calculated as discussed above. The Void peak elicits a slight fluorescence increase; the specific activity of this subset of $\alpha$-synuclein aggregates accounts for $2 \%$ of the total activity of the heterogeneous $\alpha$-synuclein preparation. This indicates that larger aggregation states of $\alpha$-synuclein do not possess significant permeabilizing activity, confirming earlier observations (18) and supporting the view that a small oligomeric species contributes to the cellular toxicity associated with PD (23). Monomeric $\alpha$-synuclein results in only $0.05 \%$ of the total membrane permeabilization activity, and this value may be overestimated due to light scattering caused by the high concentration of protein in the fluorescence flow cell. The inability of monomeric $\alpha$-synuclein to permeabilize vesicles has been demonstrated previously (19) and suggests that $\alpha$-synuclein must selfassociate in order to disrupt membranes. The relatively high molar activity level of dimeric $\alpha$-synuclein $(\sim 1 \%$ of total activity, $\sim 20$-fold more active than Monomer) is unexpected and merits further investigation. The predominant peak of fluorescence is elicited by the Protofibril fraction; the specific activity of this class of $\alpha$-synuclein aggregates represents $97 \%$ of the total activity measured. The results of this analysis indicate that the permeabilizing activity associated with aggregated $\alpha$-synuclein is confined nearly exclusively to a subfraction of these aggregates that contains annular species.

E46K a-Synuclein Protofibrils Permeabilize Synthetic Vesicles, but Display Reduced Activity. The analysis depicted in Figure 7 was employed to compare the relative vesicle permeabilization activities of protofibrillar WT and E46K $\alpha$-synuclein. The permeabilizing activity of the protofibril fraction from each variant was determined over a range of protein concentrations, and the analysis was repeated with several protein and PG vesicle preparations. Figure 8A presents the cumulative data, and indicates that although soluble oligomeric E46K $\alpha$-synuclein permeabilizes phospholipid vesicles, it does so at a lower activity level than that of the WT protein. The activity of each variant is dosedependent and saturates at a roughly similar protein concentration. Each variant displays a distinct peak permeabilization level, which indicates that the availability of intact vesicles is not a general limitation of the assay. It is possible that the saturability of the activity is the result of protofibril self-association at higher concentrations. Figure $8 \mathrm{~B}$ presents the average molar activity values (permeabilization activity per mole of total protein within the Protofibril peak) derived from E46K or WT $\alpha$-synuclein samples with relative protofibril concentration values $<20$ (the presaturation range), and confirms that the difference in activity between WT and E46K $\alpha$-synuclein soluble oligomeric species is significant $(p<0.001$ in an unpaired Student's $t$ test). Protofibrils generated from an equimolar mixture of WT and E46K monomeric protein display an average molar activity intermediate to each pure solution, indicating that the mixture results in no apparent synergistic effects. It is important to note that this analysis is unable to distinguish between a difference in individual mutant and WT protofibrils (the mutation modifies each "pore") and variation in the population of oligomeric species generated (the mutation alters the proportion of "pores" within the oligomeric mixture). Further separation of oligomeric species would be required to discriminate between these alternatives. Figure $8 \mathrm{C}$ illustrates the significantly greater average yield of protofibrils generated in vitro from WT $\alpha$-synuclein as compared with the E46K variant. Given the increased propensity of the E46K variant to form fibrils, it is possible that a greater proportion of the oligomeric material formed from this variant is of a higher order than that formed by WT $\alpha$-synuclein and is removed during processing of the protofibril-enriched preparations due to a decrease in solubility and/or increased retention during $0.2 \mu \mathrm{m}$ filtration.

\section{DISCUSSION}

Compelling evidence links $\alpha$-synuclein to the pathogenic process that underlies PD. Aggregation of the protein appears to play a central role in the disease, as indicated in part by 
A

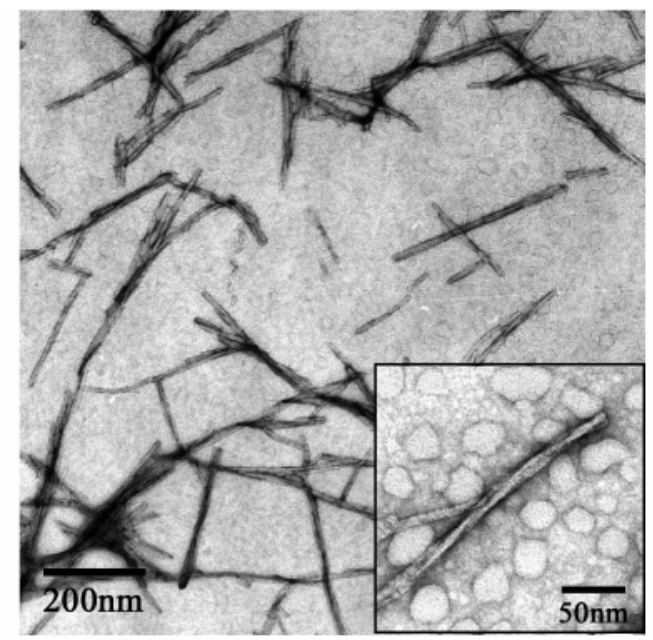

E46K
B

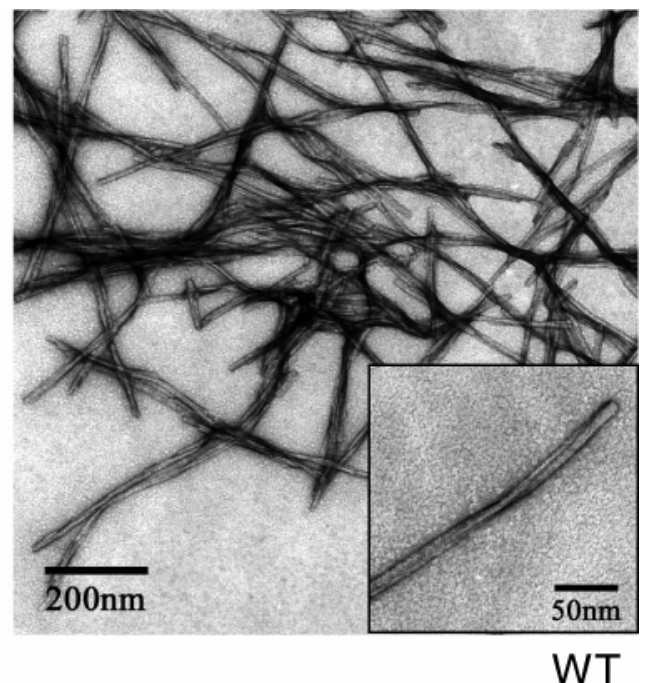

FIGURE 5: Electron micrographs illustrating the similar ultrastructural characteristics of WT and E46K $\alpha$-synuclein fibrils formed in vitro. (A) E46K and (B) WT $\alpha$-synuclein fibrils recorded at $20000 \times$. Inset images at $60000 \times$ display the morphology of single fibrils.

A

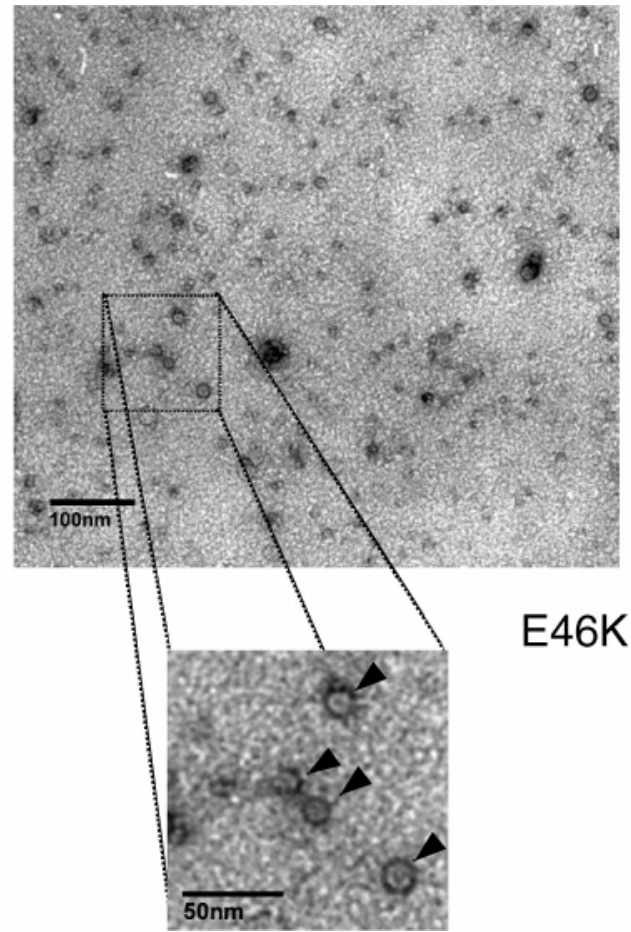

B

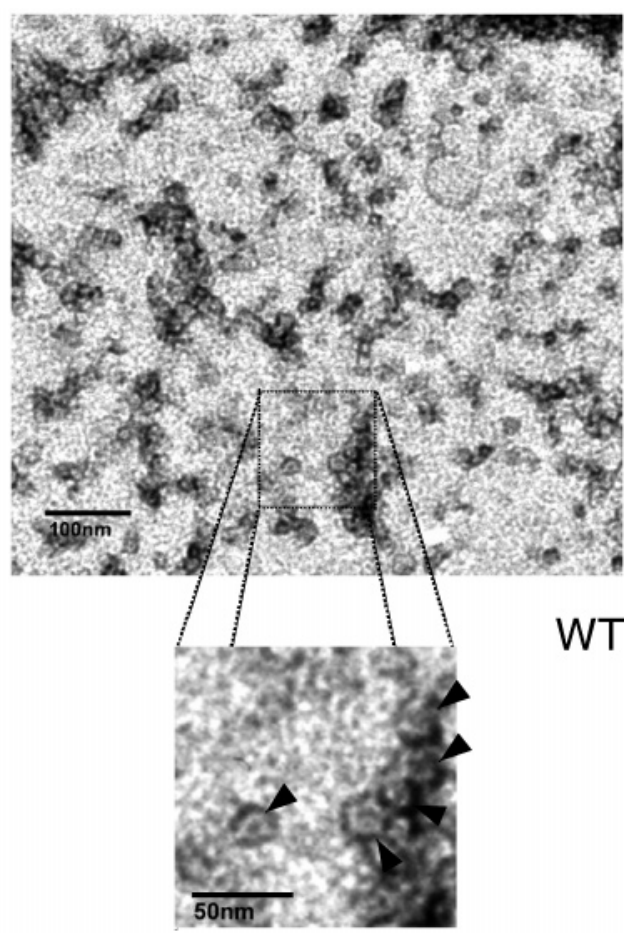

FIGURE 6: Comparison of the conformation of $\alpha$-synuclein oligomeric species by electron microscopy. (A) E46K and (B) WT $\alpha$-synuclein soluble oligomeric fractions examined at $25000 \times$ and $30000 \times$, respectively (please note that the magnification levels have been digitally normalized to facilitate comparison of the images). Magnified region illustrates the appearance of annular structures within the solution (arrows).

the appearance of $\alpha$-synuclein fibrils in Lewy bodies within the human midbrain (8), the development of $\alpha$-synuclein inclusions in various animal models of PD $(41-44)$, and the apparent conversion of $\alpha$-synuclein from a neuroprotective agent at low concentrations to a neurotoxic agent at high concentrations $(7,45)$. The contribution of a loss of normal $\alpha$-synuclein function to the disease phenotype is unclear. Although evaluation of $\alpha$-synuclein knock-out mice revealed only minor neurotransmission deficits (46), a recent study has demonstrated that $\alpha$-synuclein functions in a redundant manner with $\mathrm{Csp} \alpha$, a presynaptic protein involved in SNARE protein recycling (7). Loss of normal levels of soluble and fully functional $\alpha$-synuclein may expose the cell to increased susceptibility to stress, and therefore the effect of sequence modification on the soluble monomeric form of $\alpha$-synuclein is important to consider in addition to effects of mutation on the aggregation of the protein.

Insight into the molecular mechanism by which $\alpha$-synuclein aggregation may mediate neurotoxicity has been gleaned from biophysical and biochemical characterization of WT $\alpha$-synuclein and the familial PD (FPD) $\alpha$-synuclein variants $\mathrm{A} 53 \mathrm{~T}$ and $\mathrm{A} 30 \mathrm{P}$. Accelerated fibrillization rate is not a shared property of the A53T and A30P $\alpha$-synuclein variants, suggesting that an increase in fibrillization rate is not required for an increase in toxicity (10). However, the formation of intermediate aggregates (protofibrils) is enhanced by both mutations in comparison to the WT protein (10). Although the existence of fibrillar LBs serves as the 


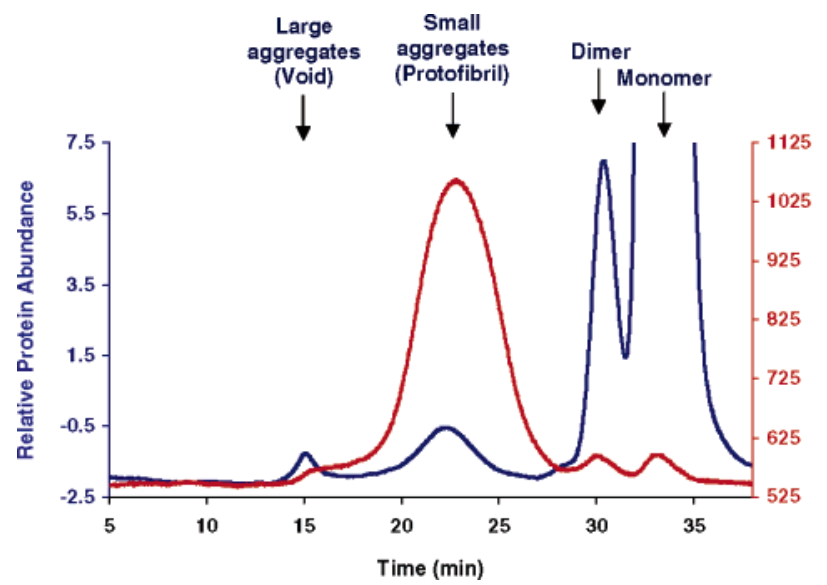

FIGURE 7: Vesicle permeabilization activity is confined to a specific subset of soluble oligomeric species of $\alpha$-synuclein. Shown is an overlay of chromatograms generated from the eluate from a Superose 6 SEC column after injection of a WT $\alpha$-synuclein preparation enriched in oligomeric species. Protein content (blue trace) was monitored at $280 \mathrm{~nm}$. Fluorescence increase due to fura2-loaded PG vesicle permeabilization (red trace) was monitored at $500 \mathrm{~nm}$ (excitation at $334 \mathrm{~nm}$ ). Note that the fluorescence trace has been shifted 1 min to the left to account for the volume within the reaction tubing between the absorbance and fluorescence detectors. The ordinate has been scaled to best observe the oligomeric forms of $\alpha$-synuclein; please note that the peak of monomeric $\alpha$-synuclein projects to a relative protein abundance level of 162 .

hallmark diagnostic criterion for PD (47), isolated $\alpha$-synuclein fibrils have proven to be relatively inert in vesicle permeabilization assays (18). In contrast, protofibrils comprising $\alpha$-synuclein demonstrate clear permeabilization activity; the FPD variants possess elevated specific permeabilizing activity compared to the WT protein (19). The existence of porelike elements within the array of soluble aggregates formed by $\alpha$-synuclein complements this functional data (16, 17,40 ); based on these findings, the protofibril hypothesis for $\alpha$-synuclein-mediated neurodegeneration in PD was proposed (23). The model predicts that a soluble, porelike intermediate to $\alpha$-synuclein fibril formation contributes to cellular toxicity, and that the relative activity of the protofibrils formed from a particular variant of $\alpha$-synuclein correlates to the relative level of toxicity created in the cell. Recent studies have supported this theory by demonstrating that a soluble oligomeric form of $\alpha$-synuclein is toxic in cell culture and animal models $(21,48-50)$.

The dynamics of E46K aggregation impact the current understanding of the role of $\alpha$-synuclein in PD pathogenesis. Recent articles have reported that E46K $\alpha$-synuclein exhibits an increased rate of fibrillization, similar to that of the A53T variant $(13,14)$. Our results confirm this finding, but extend the analysis of E46K $\alpha$-synuclein self-association to oligomeric structures that we believe have a greater predictive value in terms of potential cellular toxicity. Two points uncovered in this report provide further evidence in favor of the protofibril hypothesis. First, the E46K $\alpha$-synuclein variant generates porelike soluble aggregates and displays permeabilizing activity when in oligomeric form. These annular oligomeric species are similar in size and morphology to annular species isolated from A53T and A30P $\alpha$-synuclein as well as pathogenic $\mathrm{A} \beta$ variants $(16,40)$. The conservation of these properties among neuropathic proteins reinforces the robust nature of the phenomenon, and suggests a link between these species and neurodegeneration. Second, vesicle-permeabilizing activity has been exclusively attributed to a subfraction of oligomeric $\alpha$-synuclein that contains annular structures. This supports the view that $\alpha$-synuclein can form a distinct oligomeric species that is intrinsically disruptive to membranes; the SEC-based procedure presented here may facilitate the further isolation of protofibril activity as higher-resolution separation methods are developed.

Although E46K $\alpha$-synuclein retains properties that have been proposed to contribute to $\mathrm{PD}$ pathogenesis, it is important to note that the differences between WT, A53T, A30P, and E46K $\alpha$-synuclein warn against oversimplifying the mechanism of neurotoxicity and neglecting the potential importance of other factors that are not present in our in vitro experiments. First, while the A53T and A30P mutations both promote accumulation of protofibrils in vitro (10), the E46K mutation does not, and it in fact reduces the abundance of such aggregates. Second, the protofibrillar E46K $\alpha$-synuclein fraction displays reduced in vitro permeabilizing activity in relation to the corresponding WT oligomeric fraction; A30P and A53T $\alpha$-synuclein both possess greater activity than WT in this regard (19). Therefore, if the porelike activity of $\alpha$-synuclein is important for neurotoxicity, there must be factors in the neuronal cytoplasm that reverse the trends in the intrinsic properties of E46K versus WT $\alpha$-synuclein that are observed in vitro. For example, the target neuronal membrane will have a different composition than that of the vesicles optimized for the in vitro studies presented here and it is possible that the E46K protofibril binds to and/or permeabilizes native neuronal membranes more efficiently than does the WT protofibril. In addition, many of the properties of the neuronal cytoplasm (local pH, molecular crowding, calcium concentration, etc.) as well as cellular regulatory mechanisms (protein modification and programmed degradation) could affect E46K and WT $\alpha$-synuclein differently.

Experiments are underway to correlate in vitro permeabilizing activity with decreased cellular viability, thereby addressing the issue of the relevance of the in vitro permeabilization assay. While such experiments may also indicate the strength of the association between $\alpha$-synuclein oligomeric species and neuronal degeneration, further purification of the annular oligomeric form of $\alpha$-synuclein is necessary to provide direct evidence of a toxic protofibrillar pore. Introduction of isolated $\alpha$-synuclein pores to the cytoplasm of cultured cells could provide an indication of the ability of these species to achieve membrane permeabilization under cytoplasmic conditions. These experiments await the development of a high-resolution separation method appropriate for $\alpha$-synuclein aggregates, and may be complicated by the tenuous kinetic stability of the annular species (16). Ultimately, it is likely that purely biophysical characterization of $\alpha$-synuclein in isolation will be insufficient to establish the mechanism or mechanisms by which its different aggregate forms disrupt cellular homeostasis, as interactions within the cell may affect the ability of $\alpha$-synuclein to self-associate or perform its natural function.

The ability of monomeric $\alpha$-synuclein to bind to cellular membranes may be vital to its normal role in the cell, as evidenced by the fact that A30P $\alpha$-synuclein (a variant known to display reduced membrane binding $(34,51))$ fails 

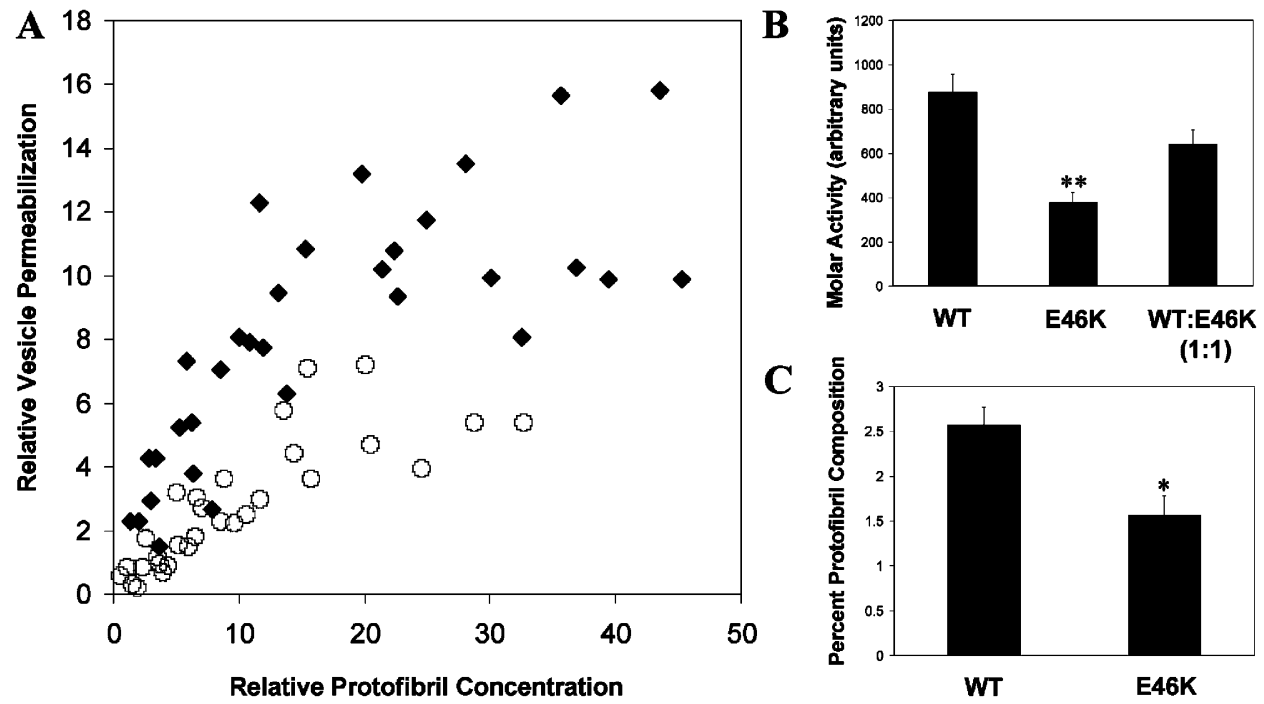

FIGURE 8: Both WT and E46K protofibrils permeabilize synthetic vesicles, but WT protofibrils display greater activity. (A) A plot of integrated peak areas of absorbance ( $x$-axis) and fluorescence ( $y$-axis) taken from Superose 6 chromatograms as shown in Figure 7. WT protofibrils, filled diamonds; E46K protofibrils, open circles. (B) The average molar activity of protofibrils generated from WT, E46K, and an equimolar mixture of both. For this analysis, molar activity is defined as the fluorescence peak area divided by the UV peak area for each point in Figure 8A within the linear portion of each curve. Error bars indicate plus or minus one standard error of 23 replicates (WT), 25 replicates (E46K), and 13 replicates (E46K:WT [1:1]). (**) indicates a $p$ value $<0.001$ when an unpaired Student's $t$ test is applied to the indicated data sets. (C) Average ratio of oligomeric $\alpha$-synuclein to the monomeric form in the WT and E46K sequence variants. Means calculated from 7 separate preparations of protofibril-enriched E46K or WT; error bars indicate plus or minus one standard error. (*) $p<$ 0.05 .

to rescue the neurotoxicity associated with the deletion of the synaptic protein $\operatorname{Csp} \alpha$ in mice (7). Here we have demonstrated that monomeric E46K $\alpha$-synuclein retains the ability to interact with PG phospholipid vesicles, as illustrated by the induction of helical character measured by $C D$. The proportion of E46K $\alpha$-synuclein molecules found in a helical state at any given lipid concentration was highly similar to that of the WT protein; this indicates that no difference in binding affinity exists between the variants. This is inconsistent with a previous report of elevated affinity of E46K $\alpha$-synuclein for synthetic synaptic vesicles (13), and may reflect the importance of lipid composition in $\alpha$-synuclein membrane association. NMR analysis of the E46K variant in its helical state reveals structural alterations within the two helical regions of the protein $(35,36)$ that propagate well beyond the local site of the mutation. While the magnitude of these changes is not large enough to suggest that the secondary structure of the protein is disrupted or abrogated by the mutation, it is quite likely that the mutation does result in some rearrangement of the helical structure with respect to its surrounding environment. Because the two helices do not contact one another $(38,52,53)$, a rearrangement of their relative positions would not be expected to result in the observed changes. Remaining possible causes include an alteration in the dynamics of the protein, a reorientation of the helices with respect to the micelle surface, or an alteration in the structure and or dynamics of the micelle itself. In the case of the A30P and A53T mutations the helical structure of micelle-bound $\alpha$-synuclein is also preserved, although the A30P mutation causes a local perturbation $(38,39)$. The A30P, but not the A53T, mutation also led to amide group chemical shift changes that extended far beyond the site of the mutation. Further study, including a full-scale structure determination for the E46K mutant protein and detailed investigations of its interactions with micelles and lipid membranes, will likely be required to clarify this issue, but these initial findings indicate that a subtle structural change in the helical region of E46K is apparent upon binding to micelles. As the lipid-bound form of $\alpha$-synuclein is believed to mediate the normal functions of the protein, the observation that the E46K mutation has long-range structural effects in the micelle-bound form of the protein suggests that this mutation may significantly influence the normal functions of $\alpha$-synuclein.

The NMR amide group chemical shift data indicate that the E46K mutation results in only very minor conformational changes in the free state of $\alpha$-synuclein, as might be expected for a largely unstructured polypeptide. Earlier studies of the A30P and A53T mutants revealed changes in secondary structure propensities in the $\mathrm{N}$-terminal lipid-binding domain of the protein, but these effects were reflected primarily in carbon chemical shifts (54). More recently several reports have used paramagnetic relaxation NMR and residual dipolar constant measurements to document a transient long-range interaction between a hydrophobic region within the $\mathrm{C}$ terminal tail of $\alpha$-synuclein and the $\mathrm{N}$-terminal lipid-binding domain, which is disrupted by the A30P and A53T mutations (55-57). This long-range interaction is not associated with chemical shift changes, and it thus remains possible that a similar effect is exerted by the E46K mutation. However, the fact that E46K $\alpha$-synuclein displays an increase in SEC retention time in comparison to the WT protein suggests that such transient interactions may be stabilized by the mutation, resulting in a more compact conformation. The relationship between the structure of monomeric $\alpha$-synuclein while free in solution and the function (or dysfunction) of the protein is currently unclear, but the results presented here indicate that further attention is warranted in this area of study.

The studies detailed above attempt to integrate the properties of the E46K variant of $\alpha$-synuclein into the current understanding of $\alpha$-synuclein-mediated toxicity through an examination of its biochemical and biophysical characteris- 
tics. The intrinsic properties of oligomeric A53T and A30P $\alpha$-synuclein, as measured by vesicle permeabilization and protofibril accumulation, may be sufficient to model the accelerated disease progression observed in FPD. It is likely, however, that extrinsic factors contribute to the toxicity of $\alpha$-synuclein in cells, and the results obtained here with E46K $\alpha$-synuclein highlight this possibility, as no clear indications of its increased neurotoxicity are evident in the inherent properties of the variant in its monomeric and oligomeric forms. The discovery and evaluation of a third disease-linked variant of $\alpha$-synuclein indicates that the current understanding of the molecular mechanism of $\alpha$-synuclein toxicity in PD is incomplete, but confirms certain aspects of the toxic protofibril hypothesis by further supporting the view that oligomeric $\alpha$-synuclein is inherently disruptive to membranes. Expanding the scope of analysis in pursuit of a detailed comprehension of the processing of $\alpha$-synuclein within the cell may lead to the identification of specific therapeutic targets and support the development of drugs to slow the progression of PD pathology.

\section{ACKNOWLEDGMENT}

The authors wish to thank Dr. Michael Volles for the development of the on-line permeabilization system and for helpful discussions during the implementation of the technique. Dr. Volles, Dr. Sarah Luchansky, and Andrew Choi provided valuable critical comments on the manuscript.

\section{SUPPORTING INFORMATION AVAILABLE}

Four figures that illustrate the purity of the protein employed in the above studies, examine the interaction of $\alpha$-synuclein with lipid vesicles, provide structural analysis of soluble oligomeric fractions of $\alpha$-synuclein from SEC, and demonstrate the consistency of the relative rates of fibril formation between WT and E46K $\alpha$-synuclein. This material is available free of charge via the Internet at http:// pubs.acs.org.

\section{REFERENCES}

1. Zarranz, J. J., Alegre, J., Gomez-Esteban, J. C., Lezcano, E., Ros, R., Ampuero, I., Vidal, L., Hoenicka, J., Rodriguez, O., Atares, B., Llorens, V., Tortosa, E. G., Del, Ser, T., Munoz, D. G., and De Yebenes, J. G. (2004) The new mutation, E46K, of alphasynuclein causes parkinson and Lewy body dementia, Ann. Neurol. $55,164-173$.

2. Valls-Sole, J., and Valldeoriola, F. (2002) Neurophysiological correlate of clinical signs in Parkinson's disease, Clin. Neurophysiol. 113, 792-805.

3. Chinta, S. J., and Andersen, J. K. (2005) Dopaminergic neurons, Int. J. Biochem. Cell Biol. 37, 942-946.

4. Polymeropoulos, M. H., Lavedan, C., Leroy, E., Ide, S. E., Dehejia, A., Dutra, A., Pike, B., Root, H., Rubenstein, J., Boyer, R., Stenroos, E. S., Chandrasekharappa, S., Athanassiadou, A., Papapetropoulos, T., Johnson, W. G., Lazzarini, A. M., Duvoisin, R. C., Di Iorio, G., Golbe, L. I., and Nussbaum, R. L. (1997) Mutation in the alpha-synuclein gene identified in families with Parkinson's disease, Science 276, 2045-2047.

5. Kruger, R., Kuhn, W., Muller, T., Woitalla, D., Graeber, M., Kosel, S., Przuntek, H., Epplen, J. T., Schols, L., and Riess, O. (1998) Ala30Pro mutation in the gene encoding alpha-synuclein in Parkinson's disease, Nat. Genet. 18, 106-108.

6. Murphy, D. D., Rueter, S. M., Trojanowski, J. Q., and Lee, V. M. (2000) Synucleins are developmentally expressed, and alphasynuclein regulates the size of the presynaptic vesicular pool in primary hippocampal neurons, J. Neurosci. 20, 3214-3220.
7. Chandra, S., Gallardo, G., Fernandez-Chacon, R., Schluter, O. M., and Sudhof, T. C. (2005) Alpha-synuclein cooperates with CSPalpha in preventing neurodegeneration, Cell 123, 383-396.

8. Spillantini, M. G., Schmidt, M. L., Lee, V. M., Trojanowski, J. Q., Jakes, R., and Goedert, M. (1997) Alpha-synuclein in Lewy bodies, Nature 388, 839-840.

9. Weinreb, P. H., Zhen, W., Poon, A. W., Conway, K. A., and Lansbury, P. T., Jr. (1996) NACP, a protein implicated in Alzheimer's disease and learning, is natively unfolded, Biochemistry 35, 13709-13715.

10. Conway, K. A., Lee, S. J., Rochet, J.-C., Ding, T. T., Williamson, R. E., and Lansbury, P. T., Jr. (2000) Acceleration of oligomerization, not fibrillization, is a shared property of both alphasynuclein mutations linked to early-onset Parkinson's disease: implications for pathogenesis and therapy, Proc. Natl. Acad. Sci. U.S.A. 97, 571-576.

11. Conway, K. A., Harper, J. D., and Lansbury, P. T., Jr. (1998) Accelerated in vitro fibril formation by a mutant alpha-synuclein linked to early-onset Parkinson disease, Nat. Med. 4, 1318-1320.

12. Li, J., Uversky, V. N., and Fink, A. L. (2001) Effect of familial Parkinson's disease point mutations A30P and A53T on the structural properties, aggregation, and fibrillation of human alphasynuclein, Biochemistry 40, 11604-11613.

13. Choi, W., Zibaee, S., Jakes, R., Serpell, L. C., Davletov, B., Anthony Crowther, R., and Goedert, M. (2004) Mutation E46K increases phospholipid binding and assembly into filaments of human alpha-synuclein, FEBS Lett. 576, 363-368.

14. Greenbaum, E. A., Graves, C. L., Mishizen-Eberz, A. J., Lupoli, M. A., Lynch, D. R., Englander, S. W., Axelsen, P. H., and Giasson, B. I. (2005) The E46K mutation in alpha-synuclein increases amyloid fibril formation, J. Biol. Chem. 280, 78007807.

15. Pandey, N., Schmidt, R. E., and Galvin, J. E. (2006) The alphasynuclein mutation E46K promotes aggregation in cultured cells, Exp. Neurol. 197, 515-520.

16. Lashuel, H., Petre, B., Wall, J., Simon, M., Nowak, R., Walz, T., and Lansbury, P. (2002) alpha-Synuclein, Especially the Parkinson's Disease-associated Mutants, Forms Pore-like Annular and Tubular Protofibrils, J. Mol. Biol. 322, 1089.

17. Ding, T. T., Lee, S. J., Rochet, J. C., and Lansbury, P. T., Jr. (2002) Annular alpha-synuclein protofibrils are produced when spherical protofibrils are incubated in solution or bound to brainderived membranes, Biochemistry 41, 10209-10217.

18. Volles, M. J., Lee, S. J., Rochet, J.-C., Shtilerman, M. D., Ding, T. T., Kessler, J. C., and Lansbury, P. T., Jr. (2001) Vesicle permeabilization by protofibrillar alpha-synuclein: implications for the pathogenesis and treatment of Parkinson's disease, Biochemistry 40, 7812-7819.

19. Volles, M. J., and Lansbury, P. T., Jr. (2002) Vesicle permeabilization by protofibrillar alpha-synuclein is sensitive to Parkinson's disease-linked mutations and occurs by a pore-like mechanism, Biochemistry 41, 4595-4602.

20. Orth, M., and Tabrizi, S. J. (2003) Models of Parkinson's disease, Movement Disord. 18, 729-737.

21. Auluck, P. K., Meulener, M. C., and Bonini, N. M. (2005) Mechanisms of Suppression of alpha-Synuclein Neurotoxicity by Geldanamycin in Drosophila, J. Biol. Chem. 280, 2873-2878.

22. Chen, L., and Feany, M. B. (2005) Alpha-synuclein phosphorylation controls neurotoxicity and inclusion formation in a Drosophila model of Parkinson disease, Nat. Neurosci. 8, 657-663.

23. Lansbury, P. T., Jr., and Brice, A. (2002) Genetics of Parkinson's disease and biochemical studies of implicated gene products, Curr. Opin. Genet. Dev. 12, 299-306.

24. Sarkar, G., and Sommer, S. S. (1990) The "megaprimer" method of site-directed mutagenesis, Biotechniques 8, 404-407.

25. Barik, S. (1995) Site-directed mutagenesis by double polymerase chain reaction, Mol. Biotechnol. 3, 1-7.

26. Tabor, S., and Richardson, C. C. (1985) A bacteriophage T7 RNA polymerase/promoter system for controlled exclusive expression of specific genes, Proc. Natl. Acad. Sci. U.S.A. 82, 1074-1078.

27. Eliezer, D., Kutluay, E., Bussell, R., Jr., and Browne, G. (2001) Conformational properties of alpha-synuclein in its free and lipidassociated states, J. Mol. Biol. 307, 1061-1073.

28. Kessler, J. C., Rochet, J. C., and Lansbury, P. T., Jr. (2003) The $\mathrm{N}$-terminal repeat domain of alpha-synuclein inhibits beta-sheet and amyloid fibril formation, Biochemistry 42, 672-678. 
29. Delaglio, F., Grzesiek, S., Vuister, G. W., Zhu, G., Pfeifer, J., and Bax, A. (1995) NMRPipe: a multidimensional spectral processing system based on UNIX pipes, J. Biomol. NMR 6, 277293.

30. Johnson, B. A., and Blevins, R. A. (1994) NMRView: A computer program for the visualization and analysis of NMR data, J. Biomol. NMR 4, 603-614.

31. Wishart, D. S., Bigam, C. G., Yao, J., Abildgaard, F., Dyson, H. J., Oldfield, E., Markley, J. L., and Sykes, B. D. (1995) 1H, 13C and $15 \mathrm{~N}$ chemical shift referencing in biomolecular NMR, $J$. Biomol. NMR 6, 135-140.

32. Conway, K. A., Harper, J. D., and Lansbury, P. T., Jr. (2000) Fibrils formed in vitro from alpha-synuclein and two mutant forms linked to Parkinson's disease are typical amyloid, Biochemistry $39,2552-2563$.

33. Volles, M. J. (2003) The aggregation and membrane permeabilizing activity of alpha-synuclein, In Biological Chemistry and Molecular Pharmacology, p 94, Harvard University, Cambridge, MA.

34. Perrin, R. J., Woods, W. S., Clayton, D. F., and George, J. M. (2000) Interaction of human alpha-Synuclein and Parkinson's disease variants with phospholipids. Structural analysis using sitedirected mutagenesis, J. Biol. Chem. 275, 34393-34398.

35. Bussell, R., Jr., and Eliezer, D. (2003) A structural and functional role for 11-mer repeats in alpha-synuclein and other exchangeable lipid binding proteins, J. Mol. Biol. 329, 763-778.

36. Chandra, S., Chen, X., Rizo, J., Jahn, R., and Sudhof, T. C. (2003) A broken alpha-helix in folded alpha-Synuclein, J. Biol. Chem. 278, 15313-15318.

37. Ulmer, T. S., Bax, A., Cole, N. B., and Nussbaum, R. L. (2005) Structure and dynamics of micelle-bound human alpha-synuclein, J. Biol. Chem. 280, 9595-9603.

38. Ulmer, T. S., and Bax, A. (2005) Comparison of structure and dynamics of micelle-bound human alpha-synuclein and Parkinson disease variants, J. Biol. Chem. 280, 43179-43187.

39. Bussell, R., Jr., and Eliezer, D. (2004) Effects of Parkinson's disease-linked mutations on the structure of lipid-associated alphasynuclein, Biochemistry 43, 4810-4818.

40. Lashuel, H. A., Hartley, D., Petre, B. M., Walz, T., and Lansbury, P. T., Jr. (2002) Neurodegenerative disease: amyloid pores from pathogenic mutations, Nature 418, 291.

41. Betarbet, R., Sherer, T. B., MacKenzie, G., Garcia-Osuna, M., Panov, A. V., and Greenamyre, J. T. (2000) Chronic systemic pesticide exposure reproduces features of Parkinson's disease, Nat. Neurosci. 3, 1301-1306.

42. Feany, M. B., and Bender, W. W. (2000) A Drosophila model of Parkinson's disease, Nature 404, 394-398.

43. Masliah, E., Rockenstein, E., Veinbergs, I., Mallory, M., Hashimoto, M., Takeda, A., Sagara, Y., Sisk, A., and Mucke, L. (2000) Dopaminergic loss and inclusion body formation in alphasynuclein mice: implications for neurodegenerative disorders, Science 287, 1265-1269.

44. Giasson, B. I., Duda, J. E., Quinn, S. M., Zhang, B., Trojanowski, J. Q., and Lee, V. M. (2002) Neuronal alpha-synucleinopathy with severe movement disorder in mice expressing A53T human alphasynuclein, Neuron 34, 521-533.

45. Seo, J. H., Rah, J. C., Choi, S. H., Shin, J. K., Min, K., Kim, H. S., Park, C. H., Kim, S., Kim, E. M., Lee, S. H., Lee, S., Suh, S.
W., and Suh, Y. H. (2002) Alpha-synuclein regulates neuronal survival via Bcl-2 family expression and PI3/Akt kinase pathway, FASEB J. 16, 1826-1828.

46. Abeliovich, A., Schmitz, Y., Farinas, I., Choi-Lundberg, D., Ho, W. H., Castillo, P. E., Shinsky, N., Verdugo, J. M., Armanini, M., Ryan, A., Hynes, M., Phillips, H., Sulzer, D., and Rosenthal, A. (2000) Mice lacking alpha-synuclein display functional deficits in the nigrostriatal dopamine system, Neuron 25, 239-252.

47. Eriksen, J. L., Wszolek, Z., and Petrucelli, L. (2005) Molecular pathogenesis of Parkinson disease, Arch. Neurol. 62, 353-357.

48. Lee, H. J., Khoshaghideh, F., Patel, S., and Lee, S. J. (2004) Clearance of alpha-synuclein oligomeric intermediates via the lysosomal degradation pathway, J. Neurosci. 24, 1888-1896.

49. Volles, M. J., and Lansbury, P. T., Jr. (2003) Zeroing in on the pathogenic form of alpha-synuclein and its mechanism of neurotoxicity in Parkinson's disease, Biochemistry 42, 7871-7878.

50. Pountney, D. L., Lowe, R., Quilty, M., Vickers, J. C., Voelcker, N. H., and Gai, W. P. (2004) Annular alpha-synuclein species from purified multiple system atrophy inclusions, J. Neurochem. 90, 502-512.

51. Jensen, P. H., Nielsen, M. S., Jakes, R., Dotti, C. G., and Goedert, M. (1998) Binding of alpha-synuclein to brain vesicles is abolished by familial Parkinson's disease mutation, J. Biol. Chem. 273, 26292-26294.

52. Bussell, R., Jr., Ramlall, T. F., and Eliezer, D. (2005) Helix periodicity, topology, and dynamics of membrane-associated alpha-synuclein, Protein Sci. 14, 862-872.

53. Borbat, P., Ramlall, T. F., Freed, J. H., and Eliezer, D. (2006) Inter-helix distances in lysophospholipid micelle-bound alphasynuclein from pulsed ESR measurements, J. Am. Chem. Soc. 128, 10004-10005.

54. Bussell, R., Jr., and Eliezer, D. (2001) Residual Structure and Dynamics in Parkinson's Disease-associated Mutants of alphaSynuclein, J. Biol. Chem. 276, 45996-46003.

55. Dedmon, M. M., Lindorff-Larsen, K., Christodoulou, J., Vendruscolo, M., and Dobson, C. M. (2005) Mapping long-range interactions in alpha-synuclein using spin-label NMR and ensemble molecular dynamics simulations, J. Am. Chem. Soc. 127, 476-477.

56. Bertoncini, C. W., Jung, Y. S., Fernandez, C. O., Hoyer, W., Griesinger, C., Jovin, T. M., and Zweckstetter, M. (2005) Release of long-range tertiary interactions potentiates aggregation of natively unstructured alpha-synuclein, Proc. Natl. Acad. Sci. U.S.A. 102, 1430-1435.

57. Bernado, P., Bertoncini, C. W., Griesinger, C., Zweckstetter, M., and Blackledge, M. (2005) Defining long-range order and local disorder in native alpha-synuclein using residual dipolar couplings, J. Am. Chem. Soc. 127, 17968-17969.

58. Grzesiek, S., Bax, A., Clore, G. M., Gronenborn, A. M., Hu, J. S., Kaufman, J., Palmer, I., Stahl, S. J., and Wingfield, P. T. (1996) The solution structure of HIV-1 Nef reveals an unexpected fold and permits delineation of the binding surface for the $\mathrm{SH} 3$ domain of Hck tyrosine protein kinase, Nat. Struct. Biol. 3, 340-345. 